\title{
PERMANENT MAGNET ION PROFILE MONITOR AT THE FERMILAB MAIN INJECTOR*
}

\author{
J. R. Zagel", D. J. Harding, B. C. Brown, H. D. Glass, L. R. Kozien, S. M. Pruss, J. T. Volk, \\ FNAL, Batavia, IL 60510, USA
}

\begin{abstract}
An ionization profile monitor can collect either ions or electrons produced from residual gas ionization. A magnetic field can be used to confine the drift path of the electrons, reducing distortion introduced into the clearing electric field by the beam space charge. We have designed, built, measured, and installed in the Fermilab Main Injector a permanent magnet for this purpose. We discuss the requirements for such a magnet, especially the effect of magnetic strength and imperfections on the measured electron profiles, and the performance of the magnet as built.
\end{abstract}

\section{EXISTING INSTRUMENTS}

The existing Ionization Profile Monitors (IPM) installed in Main Injector collect positive ions utilizing a $30 \mathrm{kV}$ clearing field. These systems have been previously reported [1]. Although profiles of injected beam at $8 \mathrm{Gev}$ are accurately represented, evidence of enlarged sigma at energies approaching $150 \mathrm{Gev}$ lead to the development of the instrument discussed here. Additional work using permanent magnets for an Ionization Profile Monitor was accomplished at Brookhaven National Laboratory [2].

\section{NEW DESIGN}

The design constraints for the Fermilab Main Injector allow for an unobstructed 3" square aperture. This will allow a single design to be used for either the horizontal or vertical plane. The overall size constraint was set for the eventual use of this instrument in the Fermilab Tevatron where the beam pipe is only 10 " above the floor.

The vacuum requirement was held at a maximum of $1 * 10^{-7}$ Torr. PolyEtherEtherKetone (PEEK) was used to replace Rexolite, offering lower outgassing rates, and yielding a design capable of $4.5 * 10^{-8}$ Torr. To reduce the noise produced by secondary electrons resulting from incident positive ions, a screen grid was added to the high voltage plate. Another significant change was the reconfiguration of signal collection components. All of these internal components are attached to the flange and can be easily removed from the rest of the vacuum housing for maintenance. A "C" magnet was designed to incorporate this feature.

\footnotetext{
*Work supported by the U. S. Department of Energy under Contract No. DE-AC02-76CH03000

\#zagel@fnal.gov
}

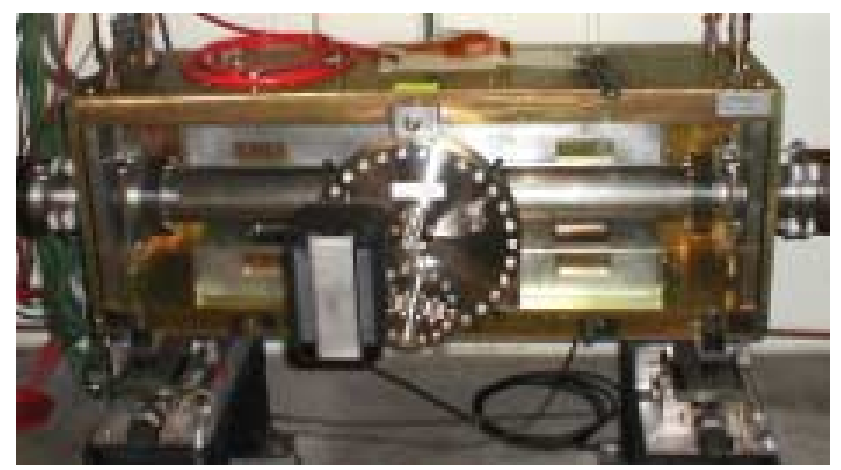

Figure 1: The system installed in the Main Injector.

The magnet design consists of 416 rare earth Samarium Cobalt $\left(\mathrm{SmCo}_{5}\right)$ permanent magnet bricks, 3 sets of steel pole pieces, and a polycarbonate cover on the open end to keep debris out. The instrument's overall dimensions are 18.5" tall (excluding stands, which are customized for each accelerator and installation), 30" from back edge of magnet to ion pump protruding from the front flange (the magnet is only 14" wide), and 49" long (78" including vacuum spool pieces.) Motorized stands were added to align the magnet remotely. The assembly can be moved linearly across the beam line and its skew can adjusted using the measurements with beam for a minimum sigma. This positioning system has 18 times the precision necessary to align within $0.5 \mathrm{~mm}$ over $10 \mathrm{~cm}$.

\section{PERMANENT MAGNET}

The IPM dipole was designed using PANDIRA supplied by the Los Alamos Code group. To achieve the desired field of 0.38 Tesla [3], $\mathrm{SmCo}_{5}$ permanent magnets were used. This material was chosen to give the highest residual magnetic field $(\mathrm{Br})$ with the lowest variation in temperature dependence. A standard brick is 1 " by 2 " by $1 / 2 "$.

Poles were made from hot rolled A36 carbon steel. Various models had shaped pole tips to reduce the higher order harmonics. However, no shaping was used since these harmonics were small enough to be ignored.

The total integrated field was designed to be zero. This was done by making the central 12 " of the pole with the field pointing up. The outer $6 "$ of the poles had the field pointing down. In between the center and end section of the poles were bucking bricks that made the transition in the field smooth. 


\section{MAGNETIC MEASUREMENTS}

The magnetic field must satisfy three main requirements. It must be sufficiently strong to contain the electrons in a tight enough helix that the resolution is not degraded. The total integrated field in the magnet should be close enough to zero to introduce minimal perturbation to the beam. And the field should be sufficiently uniform for the flux lines to remain reasonably parallel and perpendicular to the plane of the detector that there is no distortion of the electron path.

Measurements were made using a 3-axis Group-3 Hall probe. Probe movement and data acquisition were under the control of a standard Magnet Test Facility computer program. The $+\mathrm{z}$ direction is the beam axis, $+y$ is up, and $+\mathrm{x}$ is chosen to give a right-handed coordinate system. One set of measurements scanned the z-profile of the magnet in 0.2" steps; this measurement was used to determine the integrated strength. Another series of measurements performed a scan over a \pm 2 " grid in the $\mathrm{x}-\mathrm{Z}$ plane with 0.4 " step size. These scans were done at various y positions, from somewhat above the nominal beam height to below the height of the micro channel plate. These measurements were used for determining field uniformity.

\subsection{Strength}

The purpose of the magnetic field is to keep the radius of the electrons' helical path tight enough to preserve the system's spatial resolution characterized by the $0.5 \mathrm{~mm}$ anode spacing $(0.25 \mathrm{~mm}$ in the Tevatron). The measured field on the beam axis is $0.0996 \mathrm{~T}$, compared to a design value of $0.3 \mathrm{~T}$. Based on Monte Carlo simulations by Alan Hahn [4] combining the distribution of production energy and angle with the electric and magnetic field produced by the beam, it appears that this will be adequate but not generous for the Main Injector. A stronger field will definitely be required for use in the Tevatron. There is some indication that the initial calculations were not sufficiently detailed, but there is still unexplained missing flux, perhaps attributable to incompletely magnetized bricks and loss in field from the end and side.

\subsection{Integrated Strength}

To prevent the magnetic field from unacceptably disrupting the circulating beam, the main field covering the active region of the detector is compensated by regions upstream and downstream of the same strength but opposite direction and half the length. Numerically integrating the measured field points along the beam axis gives a net integrated strength of $-0.0018 \mathrm{~T}-\mathrm{m}$. Compared to the design trim dipole corrector strength of $0.090 \mathrm{~T}-\mathrm{m}(\mathrm{H})$ and $0.036 \mathrm{~T}-\mathrm{m}(\mathrm{V})$, this appears to be satisfactory. Figure 2 shows the field strength as a function of $\mathrm{z}$ along the center line of the magnet.

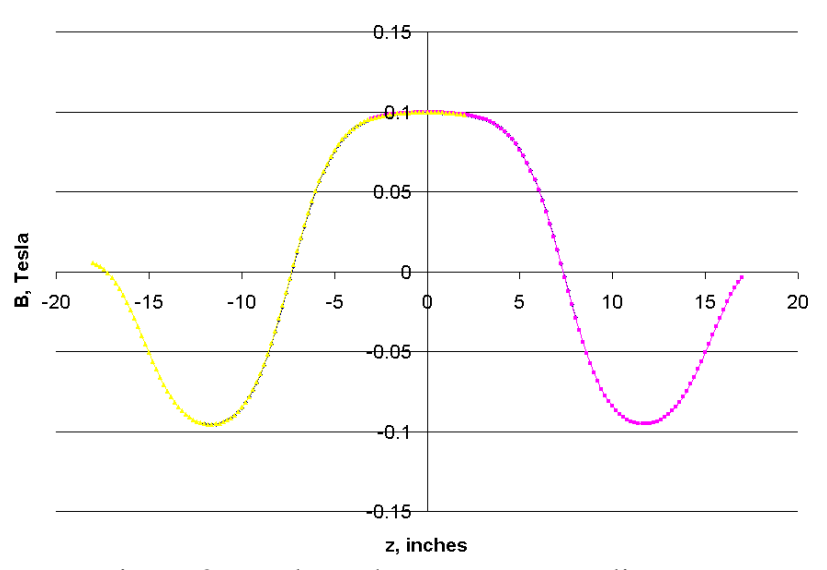

Figure 2: $\mathrm{B}_{\mathrm{y}}$ along the magnet centerline.

\subsection{Field Uniformity}

Since our picture of the electrons' behavior on its path from ionization to detector is a spiral around a "flux line", we need to extract the flux lines from the magnetic field data and assess the effect on the resolution. The raw data consists of field measurements on a rectangular grid of about $1 \mathrm{~cm}(0.4 ")$ spacing. Concentrating on a single slice in $\mathrm{z}$, a fourth order polynomial can be fit to the measured field component $\mathrm{B}_{\mathrm{y}}(\mathrm{x})$ for each $\mathrm{y}$. Typical rms agreements between fit and data are in the range 0.1 to 0.3 Gauss out of about 0.1 Tesla. The polynomial can then be integrated analytically to give the flux (per unit length in $\mathrm{z}$ ) between the magnet centerline $(\mathrm{x}=0)$ and any $\mathrm{x}$. Since our picture of the electrons' behavior is a spiral around a "flux line", we want to find values $\mathrm{x}(\mathrm{y})$ that subtend a constant flux. Taking the equally spaced measurement points on the $\mathrm{y}=0$ line as our starting points, we calculate the flux between $\mathrm{x}=0$ and each of those points. At each other value of $y$ on the grid we can then determine the value of $\mathrm{x}$ that subtends the same amount of flux. Figure 3 shows those points at $\mathrm{z}=0$, with points of constant flux connected for presentation.

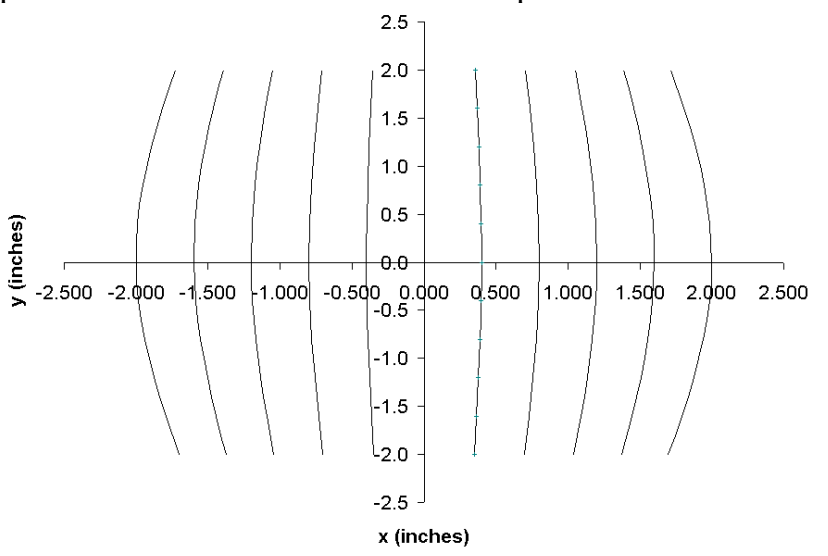

Figure 3: Flux Lines at $\mathrm{z}=0$

By inspection we see that the measured $\mathrm{x}$ of an electron varies from its production point as a function of $y$. To get the beam position in $x$ requires an independent measurement of y. Further, the intrinsic vertical spread in 
the beam size, which is integrated over by the detector, means that the width of the beam is degraded. Further, the mapping at any y will not be linear. For example, tracing the flux lines from the central orbit to the anode, we can represent the beam position as the sum of a linear plus cubic term in the observed $x$, with coefficients of 1.072 and 0.0053 (for $x$ in inches).

The field also varies with $z$. This is significant because the detector integrates in $\mathrm{z}$ and we cannot use a different mapping as we might at differing y positions. Applying the single pair of coefficients to $\mathrm{xz}$ positions results in the errors shown in Figure 4 for the reconstructed $x$ position in the plane of the central orbit. Note that the errors do vary with $\mathrm{z}$, as the compression of the flux lines varies with $\mathrm{z}$. The figure of merit is the spread among the lines, representing the values over which the detector integrates. We observe that only in the worst case, at the extremes in $\mathrm{z}$ which are beyond the active area of the detector, is the deviation greater than the $0.5 \mathrm{~mm}$ resolution of the detector.

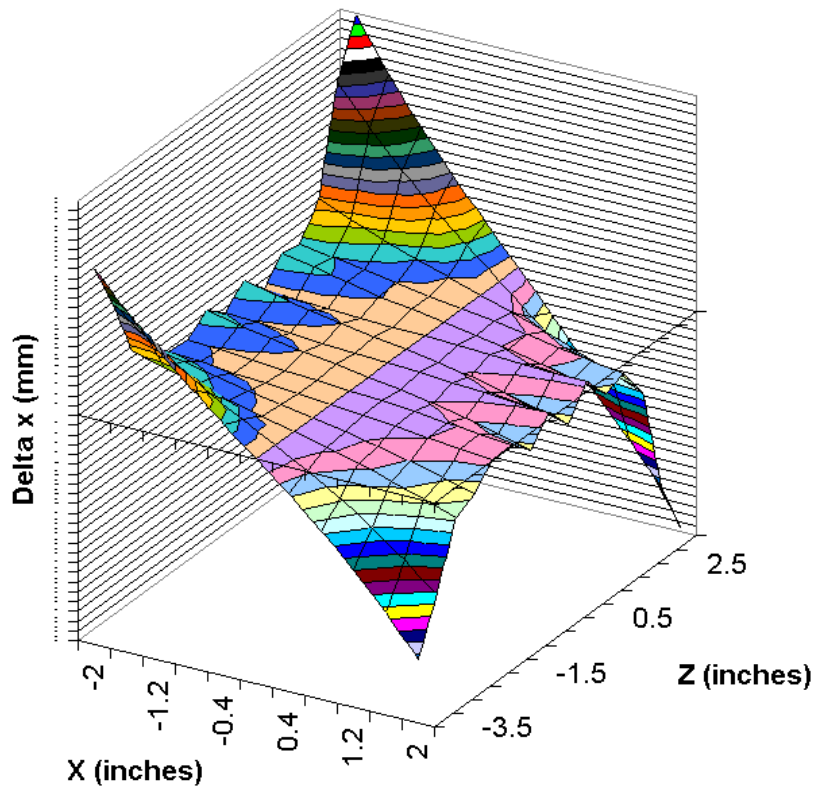

Figure 4: Difference between measured and actual $\mathrm{x} @ \mathrm{y}=0$ Contour lines are $0.2 \mathrm{~mm}$

\section{SYSTEM PERFORMANCE}

The IPM described here has been installed and tested in the Fermilab Main Injector. Turn-by-turn beam profiles have been captured, then analyzed by doing a nonlinear fit of a Gaussian to each profile. Figure 5 shows a typical display of the data collected by the IPM. On the far right is a false color intensity plot of the raw data out of the analog to digital converters. On the left are the analyzed data that have been fitted to a full nonlinear Gaussian. The top plot is a profile of the first turn of beam, showing the raw data dots and solid line fit. The middle plot shows the resulting sigma as a function of turn number while the lower plot shows the calculated mean, or position, for range of turns.

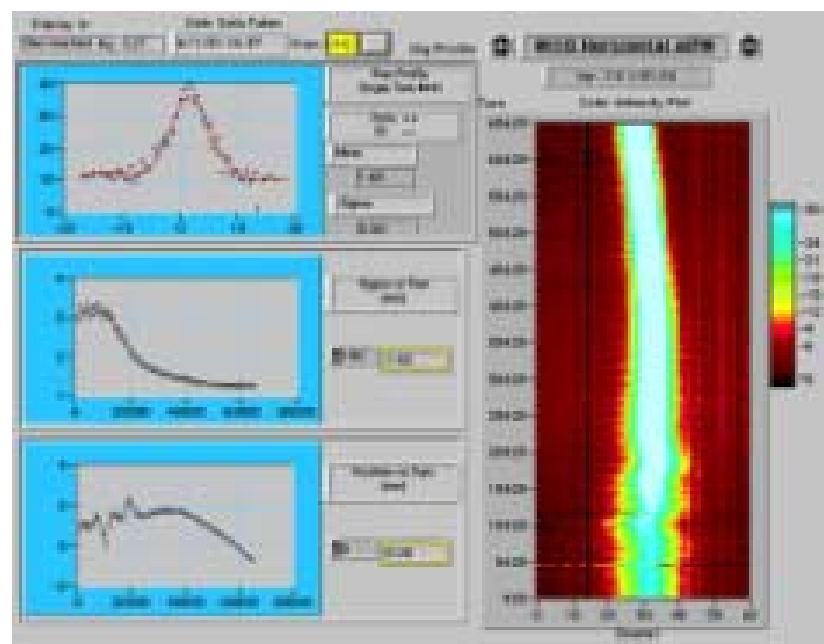

Figure 5: Sample data acquisition display.

\section{ASSESSMENT}

The electronics and the mechanical design have proved successful. The broadening of the profile is decidedly less when collecting electrons than when collecting ions. The lower field is attributed to losses from the open side and ends of the magnet not accounted for in the two dimensional model. A new version of the magnet has been modeled with the flux returns closer to the poles and side bricks used to increase the central field. It is also proposed to use Neodymium Iron Boron $(\mathrm{NdFeB})$ magnets instead of $\mathrm{SmCo}_{5}$ due to the higher energy density available. A wider pole with field-shaping shims should improve the field uniformity to the point where the beam position and width measurements are independent of position and the resolution is limited only by the anode spacing and counting statistics.

\section{ACKNOWLEDGEMENTS}

The authors would like to thank several people for their contributions. Alan Hahn's previous work lead to the eventual design and construction of this unit, and he contributed to the basic understanding of the processes involved. Mark Thompson was instrumental in gathering the magnetic measurement data. Dale Miller and Mike Frett assembled the unit. Dale also fabricated the cabling and the electronic components for data acquisition.

\section{REFERENCES}

[1] J. R. Zagel, et al., "Improvements to the Fermilab Ionization Profile Monitor Systems, PAC 99, 21642166, March 1999.

[2] P. Cameron, et al., "The RHIC Ionization Beam Profile Monitor," PAC 99, 2114-2116, March 1999.

[3] A. A. Hahn, "Tevatron IPM Note\#2," Internal Fermilab Document, October 30, 1997.

[4] Ibid 